\title{
Seasonal Treatment of an Infectious Disease is a Social Driver of Sustained Oscillations in the Disease Incidence
}

\author{
O. OSUNA ${ }^{1}$ and J. G. VILLAVICENCIO-PULIDO ${ }^{2 *}$
}

Received on September 23, 2020 / Accepted on January 19, 2021

\begin{abstract}
We analyze a seasonal SIR model that assumes a periodic treatment rate. Using the LeraySchauder degree theory, we prove that model shows periodic solutions. This result shows that sustained oscillations in the incidence of the disease are related to the periodic application of a treatment against the disease. So, we can say that the periodic application of treatment can be considered a seasonal driver of the sustained oscillations.
\end{abstract}

Keywords: seasonal treatment rate, periodic orbit, Leray-Schauder degree, SIRS models, reproduction number.

Infectious diseases have been analyzed since the Hippocratic era. In the Corpus Hippocraticum there can be found clinical descriptions of some infectious diseases. These descriptions show the relationship between the diseases and the environment, the climate, and the society. In particular, Hippocrates found a connection between the disease and the day of year when the disease appears. The first influenza epidemic of the human history was studied by Hippocrates. This disease was a winter epidemic whose infectious individuals suffered of an upper respiratory tract infection [14].

As it was pointed out above, the infectious diseases can be related to environmental or social drivers. In particular, seasonality can be considered either an environmental factor or a social factor for the spreading of infectious diseases. Seasonality is closely linked to seasonal infectious diseases; however, identifying the principal causes that produce seasonal diseases is so hard because the mechanisms producing it are little known. [1] and [5] analyze some seasonal drivers that generate a peak in the number of infectious individuals for different diseases. They found the following. Cases of vector-borne diseases as malaria and dengue have an incidence increase due to rainfall and temperature; diarrhea diseases as cholera and rotavirus infections

\footnotetext{
*Corresponding author: J. G. Villavicencio-Pulido - E-mail: j.villavicencio@correo.ler.uam.mx

${ }^{1}$ Instituto de Física y Matemáticas, Universidad Michoacana, Edif. C-3, Ciudad Universitaria, C.P. 58040. Morelia, Michoacán, México - E-mail: osvaldo@ifm.umich.mx https://orcid.org/0000-0002-4698-1321

2 Departamento de Ciencias Ambientales, Universidad Autónoma Metropolitana, Av. de las Garzas No. 10, C.P. 52005, Lerma de Villada, Estado de México, México - E-mail: j.villavicencio@correo.ler.uam.mx https://orcid.org/0000-0003$1085-8556$
} 
have a peak in the incidence because of temperature and aggregation of children; measles increases in fall or spring because the epidemiological mechanism is host aggregation during school terms; polio, rotavirus and influenza are associated to the latitude, respiratory-aerosol and contact-borne pathogens. In summary, seasonality can be related to environmental changes, host-behavior changes, seasonal change in host immune competence, and disappearance and appearance of a particular pathogen; see [1,9].

From a mathematical epidemiological perspective, periodic patterns in abiotic factors have been modelled assuming a periodic forcing function to describe the infection rate; see [6], [1], [7] and [15], [11]. In this scenario, sustained oscillation in the number of infectious individuals may occur due to a resonance phenomenon between the damped oscillations typical for SIRS models and the oscillation in the infectious rate; see [6]. For example, [6] shows that if both features share a period of one year, then the amplitude of the epidemic bouts tends to increase dramatically.

On the other hand, social drivers have been modeled to describe the dynamics of seasonal diseases. For example, [1] showed that seasonal forcing in host social behavior can generate sustained oscillations in the infected population, and ( [5], [13]) studied how yearly rhythms in host have an effect in the immune function.

In this direction, seasonal patterns are observed in the application of some public health strategies. For example, annual vaccination of susceptible individuals to protect them against the Influenza; see [10], [3]. In general, public health campaigns target susceptible individuals and the seasonal drivers are associated to the infection rate, but it is little known about how the application of a seasonal treatment to the infectious individuals affects the population dynamics.

With that in mind, in section 2 we propose a simple epidemiological model with a periodic recovery rate. In section 3 , we calculate $R_{0}$ and we prove existence of periodic orbits. In section 4 , numerical simulations of the solutions of the model are shown. Finally, in section 5, the results are discussed.

\section{THE MODEL}

We consider a population $N(t)$ that is divided into three classes: susceptible, infectious and recovered individuals, which are denoted by $S(t), I(t)$ and $R(t)$ respectively. The interactions of susceptible individuals with infectious ones are modeled using the mass action law. The infection rate of the disease, $\beta$, is assumed a positive constant, so $\beta S I$ describes the number of new infectious individuals. The natural birth/death, $\mu$, is assumed to be a positive constant. Finally, we propose a seasonal treatment rate, $\gamma(t)$; so $\gamma(t) I$ is the number of infectious individuals that are treated and recovered at time $t$. 
Then, the proposed model is

$$
\begin{aligned}
\dot{S} & =\mu(1-S)-\beta S I, \\
\dot{I} & =\beta S I-\gamma(t) I-\mu I, \\
\dot{R} & =\gamma(t) I-\mu R .
\end{aligned}
$$

Notice that, $N(t)$ is variable, with $\dot{N}=\mu-\mu N$. Then, in absence of the disease, the population goes to 1 , which is the populational carrying capacity.

By using $\dot{N}$, we can prove that the solutions of (1.1) with initial condition in the positive orthant $\mathbb{R}_{+}^{3}$ either approach, enter, or remain in the subset of $\mathbb{R}^{3}$ defined by

$$
\Sigma_{3}:=\{(S, I, R) \mid S(t) \geq 0, I(t) \geq 0, R(t) \geq 0, S(t)+I(t)+R(t) \leq 1\} .
$$

Therefore, we analyze the solutions of the model (1.1) in the invariant subset $\Sigma_{3}$.

Because the third equation of the model is independent of the first two equations in system (1.1), it can be neglected. So, we analyze the reduced model that is shown below.

$$
\begin{aligned}
& \dot{S}=\mu(1-S)-\beta S I, \\
& \dot{I}=\beta S I-\gamma(t) I-\mu I .
\end{aligned}
$$

Notice that, system (1.2) has two equilibrium points if $\gamma(t) \equiv \bar{\gamma}$ is considered constant. In this scenario, the disease-free equilibrium is given by $E_{0}=(1,0)$, and the endemic equilibrium is given by $E^{*}=\left(\frac{\bar{\gamma}+\mu}{\beta}, \mu\left(\frac{1}{\bar{\gamma}+\mu}-\frac{1}{\beta}\right)\right)$. By using the next generation matrix; see [4], we calculate the basic reproduction number that is given by $R_{0}=\frac{\beta}{\mu+\bar{\gamma}}$.

\section{$2 \overline{R_{0}}$ AND PERIODIC ORBITS FOR THE MODEL}

Knowing the evolution of an infectious disease can be possible when some epidemiological parameters are calculated. The most famous epidemiological parameter is the basic reproduction number, which is denoted by $R_{0}$. In autonomous mathematical epidemiology models, the basic reproduction number is defined as "the number of secondary infections that results from the introduction of a single infectious individual into an entirely susceptible population during its infectious period" (see [2]). By definition, if $R_{0}$ is less than 1, the number of infectious individuals will decrease; in contrast, if $R_{0}$ is greater than 1 , there is an epidemic outbreak.

However, if infectious diseases are modeled with seasonal infectious rates, secondary infections must be considered by calculating $R_{0}$. These new infections depend on the time of the year when the infectious individual is introduced into the entirely susceptible population, and an averaging of all of them is required for calculating $R_{0}$. In this case, the basic reproduction number is calculated using

$$
\bar{R}_{0}=D \int_{0}^{1} \beta(t) d t
$$


In expression (2.1), $D$ measures the average time of the infection period. So, $\bar{R}_{0}$ may be interpreted as "the average number of secondary infections that results from the introduction of a single infectious individual into a completely susceptible population at a random time of the year". In this case, the condition $\bar{R}_{0}<1$ is not enough to stop to an epidemic outbreak, yet it is sufficient and necessary for long-term disease extinction. Notice that, the control policy of bringing $\bar{R}_{0}$ below 1 fails to control the epidemic outbreak because transmission series can be created during the season of high incidence if $D \beta(t)>1$.

By proceeding in an analogous way as the one shown above, if $\beta$ is considered constant and $\gamma(t)$ is assumed a periodic solution, we propose that the basic reproduction number to be

$$
\bar{R}_{0}=D(t) \int_{0}^{1} \beta d t
$$

In this case, it is required an averaging over all the possible times of the year that an infectious individual is recovered.

For model (1.1), the basic reproduction number is

$$
R_{0}=\frac{\beta}{\bar{\gamma}+\mu}
$$

with

$$
\bar{\gamma}=\frac{1}{T} \int_{0}^{T} \gamma(t) d t
$$

The periodic rate $\gamma(t)$ can be decomposed as

$$
\gamma(t)=\bar{\gamma}+\gamma_{0}(t) \quad \text { where } \quad \int_{0}^{T} \gamma_{0}(t) d t=0 .
$$

We define the following homotopy.

$$
\begin{aligned}
& \dot{S}=\mu(1-S)-\beta S I, \\
& \dot{I}=\beta S I-\gamma_{\lambda}(t) I-\mu I,
\end{aligned}
$$

for $\lambda \in[0,1]$ and $\gamma_{\lambda}(t):=\bar{\gamma}+\lambda \gamma_{0}(t)$.

System (2.6) is an homotopy between a nonseasonal system that is given by model (2.6) with $\gamma_{0}(t)=\bar{\gamma}$ and the system (1.2), which corresponds to taking $\lambda=1$ in the model (2.6). Observe that, for $\lambda=0$, system (2.6) has the two equilibria points $E_{0}$ and $E^{*}$ that were mentioned above.

We prove existence of one nontrivial periodic solution using the Leray-Schauder degree theory; see [11]. For this, $E^{*}$ is going to continue as a function of $\lambda$ until the existence of a periodic solution for $\lambda=1$ is proved. 
Suitable modifications to describe system (1.1) will be established. To do so, the problem is formulated in a functional setting in the following way.

For $l=0,1$, the Banach spaces

$$
\mathscr{C}^{l}=\left\{(S, I) \mid S, I \in C^{l}(\mathfrak{R}, \mathfrak{R}), S(t+T)=S(t), I(t+T)=I(t)\right\}
$$

are considered.

Let $L: \mathscr{C}^{1} \rightarrow \mathscr{C}^{0}$ and $N_{\lambda}: \mathscr{C}^{0} \rightarrow \mathscr{C}^{0}$ be the operators given by

$$
L(S, I)=(\dot{S}+\mu S, \dot{I}+\mu I,)
$$

and

$$
N_{\lambda}(S, I)=\left(\mu-\beta S I, \beta S I-\gamma_{\lambda}(t) I\right)
$$

Since $L$ is invertible, the operator

$$
F_{\lambda}(S, I)=(S, I)-L^{-1} \circ N_{\lambda}(S, I)
$$

is defined.

Because $\mathscr{C}^{1}$ is complety embedded in $\mathscr{C}^{0}$ and $L^{-1}: \mathscr{C}^{0} \rightarrow \mathscr{C}^{1}$, the operator, $L^{-1} \circ N_{\lambda}: \mathscr{C}^{0} \rightarrow \mathscr{C}^{0}$ is a compact operator. In an analogous way, $F_{\lambda}: \mathscr{C}^{0} \rightarrow \mathscr{C}^{0}$ can be considered. Therefore, periodic solutions of the system (2.6) correspond to zeros of $F_{\lambda}$.

Now, consider the open sets

$$
D:=\left\{(S, I) \in \mathscr{C}^{0} \mid S(t)>0, I(t)>0, S(t)+I(t)<1\right\}
$$

and

$$
G:=\left\{(S, I) \in D \mid \min S(t)_{[0, T]}<r\right\}
$$

for a fixed $0<r<1$.

Recall that, the existence of a solution of $F_{1}$ in $G$ via Leray-Schauder degree theory is guaranteed if $\operatorname{deg}\left(F_{0}, G\right) \neq 0$, and $F_{\lambda}$ is an admissible homotopy. That is, $0 \neq F_{\lambda}(\partial G)$, for all $\lambda \in[0,1]$.

In the next result, we prove that $F_{\lambda}$ is an admissible homotopy.

Lemma 1. If $R_{0}>1$ and $r$ is such that $\frac{1}{R_{0}}<r<1$, then for any $\lambda \in[0,1]$ there are no solutions $(S, I)$ of the system (2.6) on $\partial G$.

Proof. Notice that $\left(S_{0}, I_{0}\right)$ is the unique solution of the system (2.6) on $\partial G$ for any $\lambda \in[0,1]$. If $(S, I) \in \partial G$, then $(S, I) \notin \partial D$ so $(S, I) \in D$ and

$$
S(t) \geq r, \forall t
$$


Integrating the second equation in (2.6) on the interval $[0, T]$, the expression

$$
\int_{0}^{T} \frac{\dot{I}}{I} d t=(\beta S-\mu) T-\int_{0}^{T} \gamma_{\lambda} d t
$$

is obtained. Notice that, $\int_{0}^{T} \frac{I}{I} d t=0$ because $I(t)$ is $T$-periodic. By using (2.11) and (2.12), the inequality

$$
\frac{1}{T} \int_{0}^{T} \gamma_{\lambda} d t \geq \beta S-\mu
$$

is achieved.

By using the assumptions of the Lemma (1), the expression

$$
\mu+\bar{\gamma} \geq \beta r>\beta \frac{1}{R_{0}}=\mu+\bar{\gamma}
$$

is obtained, which is a contradiction.

Therefore, Lemma (1) is proved.

Now the degree of $F_{0}$ on the open set $G$ will be determined.

Proposition 2. Let $G$ be an open set as the one defined above. Then $\operatorname{deg}\left(F_{0}, G\right) \neq 0$.

Proof. Observe that, if $R_{0}>1$, then $\left(S^{*}, I^{*}\right)$ is the unique periodic solution of $F_{0}(S, I)=0$ on $G$.

To prove that $\operatorname{deg}\left(F_{0}, G\right) \neq 0$, it suffices to show that the Fréchet derivative $D F_{0}\left(S^{*}, I^{*}\right)$ is invertible. For this, it will be proved that the kernel of $D F_{0}\left(S^{*}, I^{*}\right)$ has uniquely the trivial solution. That is, $\operatorname{ker}\left(D F_{0}\left(S^{*}, I^{*}\right)\right)=\{0\}$. This is possible because $D F_{0}\left(S^{*}, I^{*}\right)$ is Fredholm as $F$ is a compact perturbation of the identity.

Let $(V, W) \in \operatorname{ker}\left(D F_{0}\left(S^{*}, I^{*}\right)\right)$. We will prove that $\operatorname{ker}(V, W)=\{0\}$.

For this, the system $D F_{0}\left(S^{*}, I^{*}\right)=0$ must be solved, which is equivalent to solve

$$
L(V, W)=D N_{0}\left(S^{*}, I^{*}\right)(V, W) .
$$

By using equations (2.8) and (2.9), we obtain the expression

$$
D N_{0}\left(S^{*}, I^{*}\right)(V, W)=\left(-\bar{\beta}\left(S^{*} W+I^{*} V\right),\left(\bar{\beta}\left(\left(S^{*}-\bar{\gamma}\right) W+I^{*} V\right)\right) .\right.
$$

Therefore, the system (2.13) is explicitly given by

$$
\left(\begin{array}{c}
V \\
W
\end{array}\right)^{\prime}=\left(\begin{array}{cc}
\mu R_{0} & -(\bar{\gamma}+\mu) \\
\mu\left(R_{0}-1\right) & 0
\end{array}\right)\left(\begin{array}{l}
V \\
W
\end{array}\right) .
$$


The characteristic polynomial of matrix $(2.15)$ is given by

$$
p(x)=x^{2}+\mu R_{0} x+\mu(\bar{\gamma}+\mu)\left(R_{0}-1\right) .
$$

Analyzing the polynomial (2.16) is observed that the independent term $p(0)>0$ if and only if $R_{0}>1$. Also, assuming $\omega \in \mathbb{R}$,

$$
\operatorname{Im}(p(\omega i))=\mu R_{0} \omega
$$

Therefore, the matrix associated to the system (2.15) has no imaginary nor zero eigenvalues. In conclusion, the system has no periodic solutions except $(V, W)=(0,0)$. Then, the result is proved.

So, we can enunciate the principal result.

Theorem 3. Model (1.1) admits a non-trivial periodic solution if $R_{0}>1$.

Proof. Using the invariance of the Leray-Schauder degree under the homotopy, by Lemma 1 and Proposition 1, the result $\operatorname{deg}\left(F_{1}, G\right) \neq 0$ is achieved. Therefore, the system (1.1) admits a non-trivial periodic solution.

We have shown that the public health strategy given by a seasonal treatment can be considered as a factor producing sustained oscillations in the infected population still in the case that the infectious rate is assumed constant.

\section{SIMULATIONS AND NUMERICAL ANALYSIS}

In this section, numerical simulations of the models solutions are shown. For this, the software Python 3.7.3 is used. In particular, the effects of the seasonal recovery rate over the solutions of the model are shown. To do this, we propose the following function.

$$
\gamma(t)=\gamma\left(1+\imath \cos \left(\frac{2 \pi t}{T}\right)\right) .
$$

The values of the parameters used in the numerical simulations do not describe neither epidemiological rates nor demographic ones. They only were chosen such that satisfy that $R_{0}>1$ to illustrate the results of the analysis of the model.

Figure 1 and 2 show the dynamics of the epidemiological classes if the values of the parameters of the seasonal treatment rate are changed.

\section{DISCUSSION}

The epidemiological mathematical literature is vast. From the Hippocratic era, infectious diseases have been associated to social or environmental drivers. Most epidemiological models describing seasonal diseases use seasonally-dependent transmission rates to describe them. In particular, most models assume that seasonality is related to environmental factors, and in a fewer cases, 


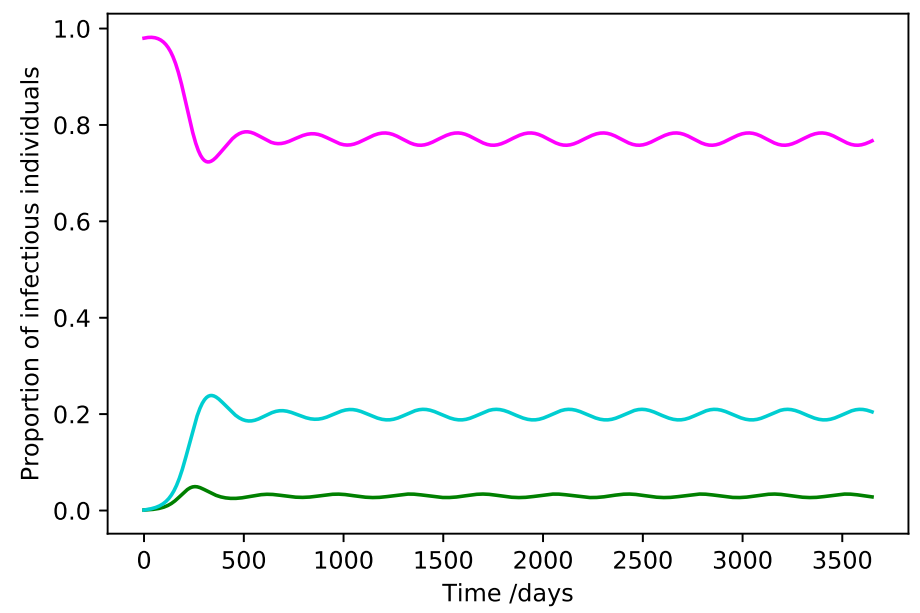

Figure 1: Figure shows sustained oscillations in the epidemiological classes. The values of the parameters of the model are $\mu=0.01, \beta=9.79 \times 10^{-2}, \gamma=0.0654554132, \imath=0.02$.

it is related to social drivers. Indeed, the study of periodic recovery rates has been avoided. Epidemiological models have been used to design public health strategies. The classical strategy is to bringing $R_{0}$ below 1 to control de infectious disease. It is important to underline that $R_{0}$ must capture the seasonality when this characteristic of the disease is modeled. Notice that, if $R_{0}$ does not capture the seasonal phenomenon, the control interventions may fail, which can be catastrophic for the population.

Decision makers take into account to design control interventions whether new infectious cases are associated with social or environmental drivers. In this sense, as it was previously mentioned, seasonal behaviors are commonly associated to periodic infection rates. Therefore, the control interventions are designed using this periodicity.

In this work, we show that sustained oscillations in the incidence of infectious individuals appear if a seasonal treatment is assumed. In other words, periodic treatments lead to seasonal behavior in the incidence of the disease that can be an undesirable scenario for the population. We calculate the basic reproduction number, $R_{0}$, which can be used to design intervention control. The analysis of a model with a seasonal treatment rate is in line with the reality because seasonal treatments are applied for seasonal infectious diseases, for example, the periodic mass administration of antibiotics for ocular Chlamydia trachomatis, and the use of antiviral therapy in person whom influenza develops; see [12] and [8].

It is known that a SIR model with a periodic infection rate shows sustained oscillation in the number of infectious individuals. In this direction, we show a complementary result. So, seasonal behavior in the incidence of the disease can be related not only to periodic infection rates but also to periodic recovery rates. Therefore, decision makers can design the public health strategies 


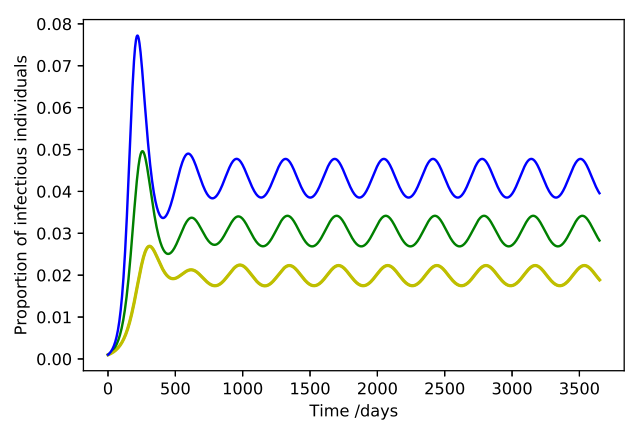

(a)

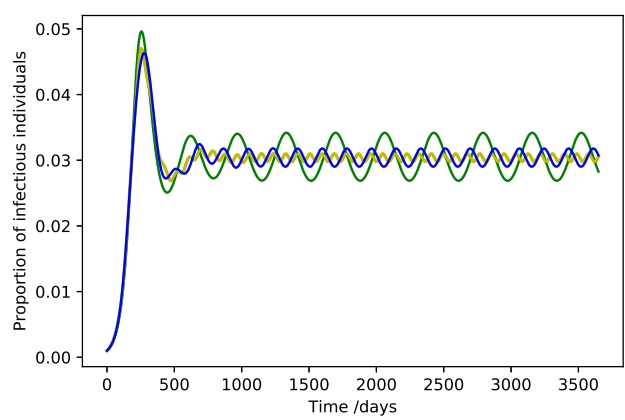

(c)

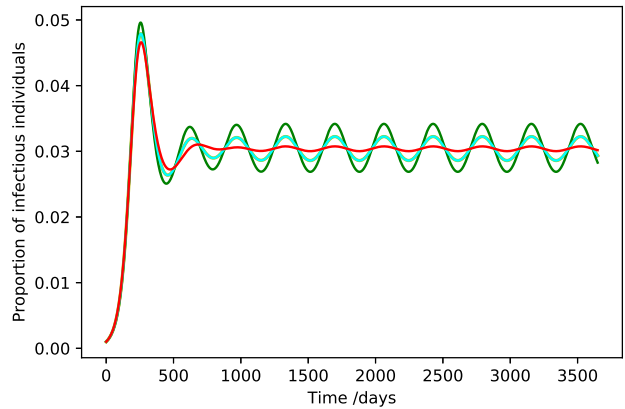

(b)

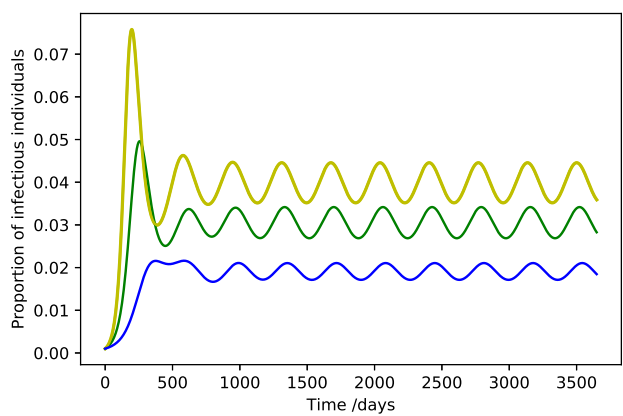

(d)

Figure 2: Case (a) shows the behavior of the infectious class if the value of the parameter $\gamma$ is changed and the other values of the parameters are the same as the values used in Figure 1. In this scenario, for the green solution $\gamma=0.0654554132$ while for the blue and yellow solutions the value of $\gamma$ is reduced and increased in $10 \%$, respectively. Case (b) shows the behavior of the infectious class if the value of the parameter $l$ is changed and the other values of the parameters are the same as the values used in Figure 1. In this scenario, for the green solution $\gamma=0.02$ while for the red and cyan solutions the value of $\imath=0.1,0.5$ is reduced $90 \%$ and $50 \%$, respectively. Case (c) shows the behavior of the infectious class if the length of the period is changed. In this scenario, for the green solution the period is 365 days, for the yellow solution the period is 90 days, and for the blue solution the period is 183 days. Finally, Case (d) shows the infectious class if the infectious rate is changed. The green solution is obtained if $\beta=9.79 \times 10^{-2}$, the yellow solution is obtained if $\beta$ is increased in $10 \%$ and the blue solution is obtained if $\beta$ is decreased in $10 \%$. 
taking into account that seasonal behavior can be associated either with seasonal infection rates or with seasonal recovery rates.

\section{Acknowledgments}

We would like to thank the anonymous referee for the careful reading of our manuscript and for providing us with constructive comments, which helped to improve the manuscript.

\section{REFERENCES}

[1] S. Altizer, A. Dobson, P. Hosseini, P. Hudson, M. Pascual \& P. Rohani. Seasonal and the dynamics of infectious diseases. Ecology Letters, 9 (2006), 467-484.

[2] R.M. Anderson \& R.M. May. "Infectious diseases of humans: dynamics and control". Oxford University Press, Oxford (1991).

[3] R.H. Borse, S.S. Shrestha, A.E. Fiore, C.Y. Atkins, J.A. Singleton, C. Furlow \& M.I. Meltzer. Effects of Vaccine Program against Pandemic Influenza A(H1N1) Virus, United States. Emerging Infectious Diseases, 19(3) (2013), 439-448.

[4] P.V. den Driessche \& J. Watmough. Reproduction numbers and sub-threshold endemic equilibria for compartmental models of disease transmission. Mathematical Biosciences, 180 (2002), 29-48.

[5] S.F. Dowell. Seasonal variation in host susceptibility and cycles of certain infectious diseases. Emerg. Infect, 7 (2001), 369-373.

[6] J. Dushoff, J.B. Plotkin, S.A. Levin \& D.J.D. Earn. Dynamical resonance can account for seasonality of influenza epidemics. Proc. Natl Acad. Sci. USA, 101 (2004), 16915-16916.

[7] D. Fishman. Seasonality of Infectious Diseases. Ann. Rev. Public Health, 28 (2007), 127-143.

[8] W.P. Glezen. Prevention and treatment of seasonal influenza. N. Engl. Med., 359 (2008), 2579-2585.

[9] N.C. Grassly \& C. Fraser. Seasonal infectious diseases epidemiology. Proceedings of the Royal Society $B, 273$ (2006), 2541-2550.

[10] Institute of Medicine (US) Forum on Microbial Threats. "The Threat of Pandemic Influenza: Are We Ready?". National Academies Press, Washington (DC) (2005).

[11] G. Katriel. Existence of periodic solutions for the periodically forced SIR model. Journal of Mathematical Sciences, 201,3 (2014), 335-342.

[12] D.C. Lee, J.D. Chidambaram, T.C. Porco \& T.M. Lietman. Seasonal effects in the elimination of trachoma. Am. J. Trop. Med. Hyg., 72(4) (2005), 468-470.

[13] R.J. Nelson, G.E. Demas, S.L. Kelin \& L.J. Kriegsfeld. "Seasonal patterns of stress, immune function and disease". Cambridge University Press, New York (2002).

[14] G. Pappas, I.J. Kiriaze \& M.E. Falagas. Insights into infectious disease in the era of Hippocrates. International Journal of Infectious Diseases, 12 (2008), 347-350. 
[15] D.G. Williams \& C. Dye. Infectious disease persistence when transmission varies seasonally. Mathematical Biosciences, 145 (2012), 77-88.

(cc) BY 